\title{
Mechanical properties of carbon nanotube under uniaxial tensile strain
}

\author{
Kazufumi Yoneyama, Mina Maruyama, Yanlin Gao, and Susumu Okada* \\ Graduate School of Pure and Applied Sciences, University of Tsukuba, 1-1-1 Tennodai, Tsukuba, Ibaraki \\ 305-8571, Japan
}

\begin{abstract}
Based on the density functional theory with the generalized gradient approximation, we investigated the mechanical properties of single-walled carbon nanotubes (CNTs) in terms of the chiral angle and atomic vacancies under a uniaxial tensile strain. Young's modulus monotonically decreases with increasing the chiral angle from zigzag to armchair. Furthermore, defects substantially decrease the modulus for all CNTs. The critical tension where the fracture starts also depends on the chirality and defect species. The chiral $(10,4)$ CNT exhibits the highest mechanical toughness against the tension of $109 \mathrm{nN}$ at the $24 \%$ elongation, while the $(7,7)$ CNT with monovacancy exhibits the lowest toughness against the tension of $88 \mathrm{nN}$ at $11 \%$ elongation.
\end{abstract}

\section{Introduction}

Ever since the discovery of carbon nanotubes (CNTs), ${ }^{1)}$ CNTs have been keeping a premier position in fundamental and applied sciences owing to their unique structural and electronic properties. ${ }^{2)}$ One of the most fascinating aspects is that CNTs are either metals or semiconductors depending on their atomic arrangements along the circumference, ${ }^{3-5)}$ originated from the discretization condition on the conical band structure around the $\mathrm{K}$ point of graphene sheet. This unusual electronic property allows CNTs emerging materials for designing electronic devices in which nanotubes act as nanometer scale channels for electrons/holes between source and drain electrodes. For instance, it was demonstrated that semiconducting CNTs can work as field-effect transistors (FETs). ${ }^{6-10)}$ Besides the electronic properties, owing to the strong covalent $\mathrm{sp}^{2}$ network of $\mathrm{C}$ atoms, CNTs possess remarkable mechanical strength, ${ }^{11,12)}$ resulting in high thermal conductivity. ${ }^{13,14)}$ Therefore, CNTs are considered to be the constituent materials of energy harvesting devices, such as a thermoelectric devices. Furthermore, nanoscale one-dimensional spacing endows CNTs with a role of molecular container where atoms

*E-mail: sokada@comas.frsc.tsukuba.ac.jp 
and molecules are encapsulated and transform into other forms being absent in their bulk phases. ${ }^{15-22)}$

For practical applications, mechanical toughness of CNTs is the most important issue to guarantee the stable operation of devices, because the CNTs are usually applied to devices with flexibility. Furthermore, CNTs are also regarded as the constituent materials for composite application where the mechanical properties of CNTs are highly important to determine the composite performance. A recent experiment reported that the mechanical properties of isolated CNTs also depend on the CNT chirality. ${ }^{23)}$ In addition, many theoretical works elucidated that mechanical properties of CNTs depend on diameter, chirality, and structural imperfections. ${ }^{24-29)}$ Indeed, it has been established that the Young's modulus of armchair CNTs is slightly higher than that of zigzag CNTs. Also the mechanical stiffness of CNTs against the tensile strain monotonically decreases with increasing their diameters. The unified knowledge of the mechanics of CNTs with respect to the chirality and vacancies are still absent to date, being highly demanded in the field of nanoscale science and technology, although these theoretical works reported the remarkable properties of the particular CNTs. Accordingly, in this work, we aim to clarify mechanical properties of CNTs with approximately $1 \mathrm{~nm}$ diameter, in terms of their chirality and atomic defects, using the density functional theory (DFT). Our calculations demonstrated that the critical tension weakly depends on the CNT chirality and defect species. On the other hand, the mechanical toughness of CNTs substantially decreases by introducing the defects. For the armchair and chiral CNTs, small negative Poisson's ratio has been observed under the small tensile strains less than a few percent.

\section{Theoretical methods and structural model}

$\mathrm{DFT}^{30,31)}$ is adopted to investigate geometric and electronic structure of CNT with tensile strain using a STATE package. ${ }^{32)}$ The exchange-correlation energy among the interacting electrons is described by the generalized gradient approximation (GGA) with the Perdew-Burke-Ernzerhof functional form. ${ }^{33,34)}$ An ultrasoft pseudopotential was employed to describe the interaction between electrons and nuclei. ${ }^{35)}$ Valence wave functions and deficit charge density were expanded in terms of the plane-wave basis set with cutoff energies of 25 and 225 Ry, respectively. Integration along the one-dimensional Brillouin zone was carried out on $4 k$-points along the CNT direction, which gives sufficient convergence in the total energy and geometric structures of graphene related materials. ${ }^{36)}$ The atomic structure of CNTs were fully optimized until the force acting 
on each atom was less than $5 \mathrm{mRy} / \AA$.

In this work, we consider three metallic CNTs with the indexes (12,0) (zigzag), (10,4) (chiral), and (7,7) (armchair) CNTs with the diameter of approximately $1 \mathrm{~nm}$, to clarify the chirality dependence of the mechanical properties of CNTs under the tensile strain [Figs. 1(a)- 1(c)]. To give quantitative discussions on the mechanical properties of CNT and to investigate the effect of atomic defect, we took the double, single, and quadruple periodicities of conventional unit of $(12,0),(10,4)$, and $(7,7) \mathrm{CNTs}$, respectively, resulting in the similar cell parameter along CNT axis of approximately $1 \mathrm{~nm}$. For the defect, we considered monovacancy and divacancy which are incorporated into CNTs per about 1 nm [Figs. 1(d)-1(i)]. Each CNT is separated from its periodic images by $0.6 \mathrm{~nm}$ vacuum spacing for simulating an isolated CNT.

\section{Results and discussion}

Figure 1 shows optimized geometries of $(12,0)$, (10,4), and $(7,7)$ CNTs without and with vacancies. For the pristine CNTs, the optimized diameters are 0.948, 0.983, and $0.953 \mathrm{~nm}$ for $(12,0),(10,4)$, and $(7,7) \mathrm{CNTs}$, respectively. Calculated total energies of $(12,0),(10,4)$, and $(7,7)$ CNTs are 92,83 , and $88 \mathrm{meV} /$ atom, respectively, measured from that of an isolated graphene. The total energy of CNT is inversely proportional to their diameter, while is insensitive to their chiral angle [Fig. 2]. Monovacancy in $(12,0),(10,4)$, and $(7,7)$ CNTs substantially increases the total energy by $4.30,4.49$, and $4.57 \mathrm{eV}$, respectively, because of the formation of unsaturated covalent bonds at the defects [Figs. 1(d)- 1(f)]. For all cases, CNTs possess at least one C atom with twofold coordination. Formation energy of divacancy is significantly lower than that of the monovacancy, owing to the formation of pentagonal rings associated with the defect. The calculated energies are $0.96,1.22$, and $1.31 \mathrm{eV}$ for $(12,0),(10,4)$, and $(7,7)$ CNTs, respectively. Because of the larger formation energy of vacancies in the armchair CNT than the others, the defect density in the armchair CNT is expected to be lower than those in the other CNTs. Furthermore, the defect density may increase with decreasing the chiral angle.

The Young's modulus of pristine and defective CNTs is summarized in Table I. Calculated moduli are 0.741, 0.748, and 0.754 TPa for pristine (12,0), (10,4), and $(7,7)$ CNTs, respectively. The armchair CNT is the most rigid among CNTs studied here. The modulus slightly decreases with increasing the chiral angle from zigzag to armchair. Vacancies substantially decrease the modulus, irrespective of their size and 
(a)

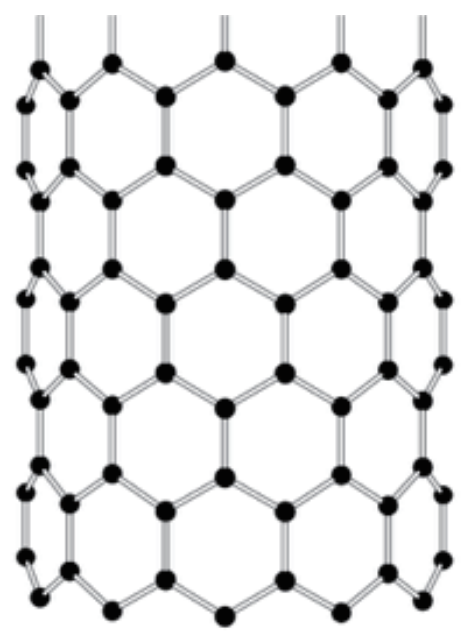

(d)

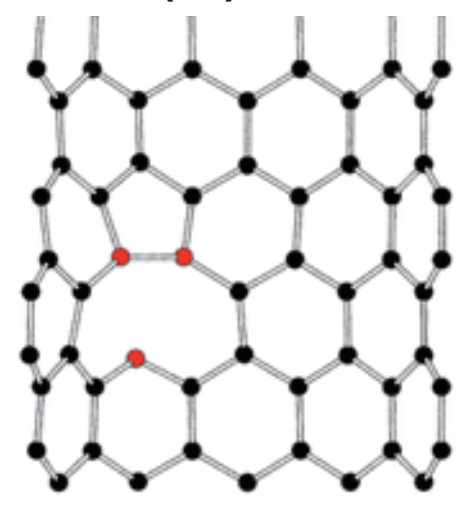

(g)

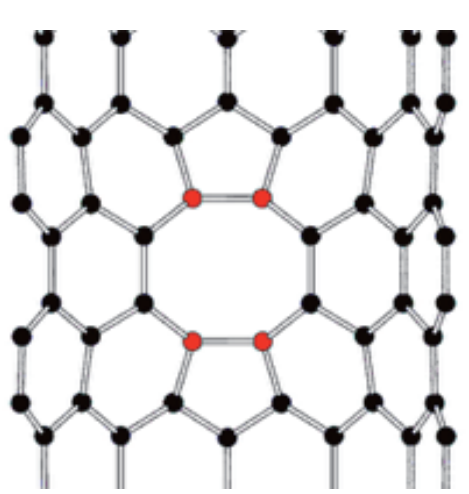

(b)

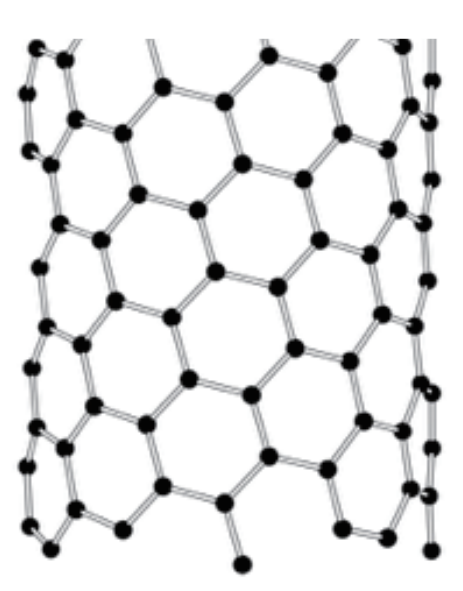

(e)

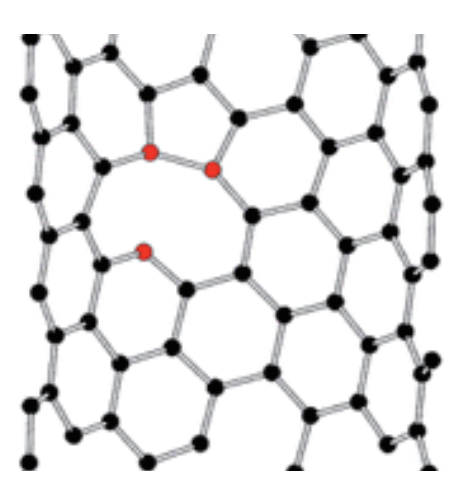

(h)

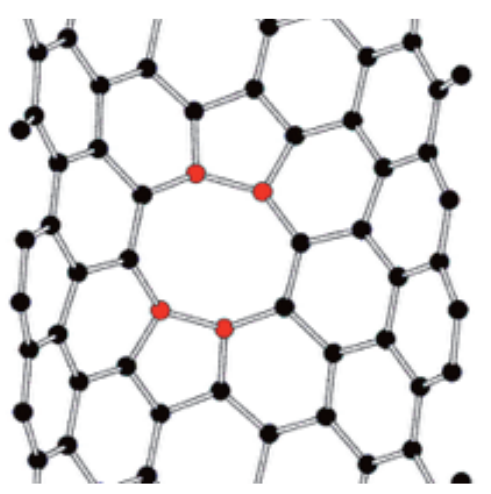

(c)

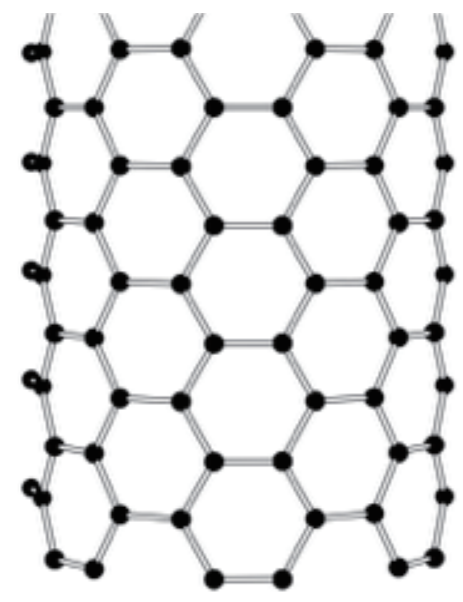

(f)

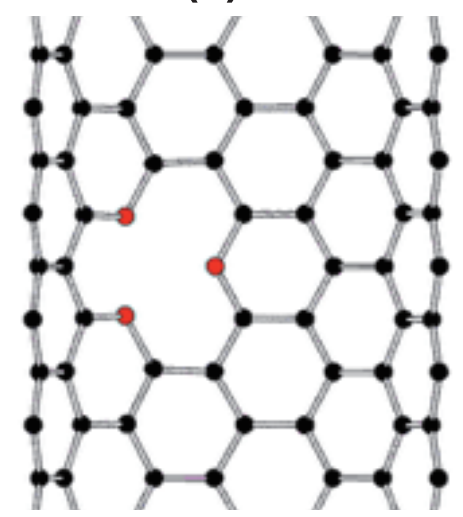

(i)

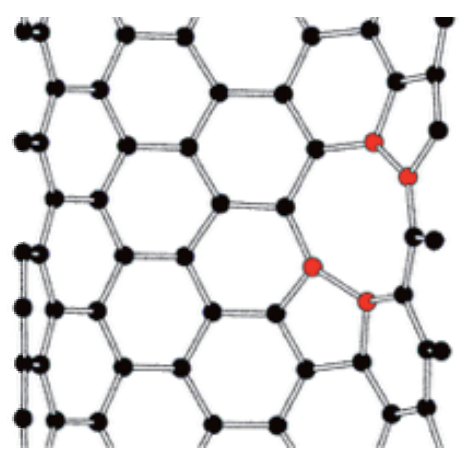

Fig. 1. Optimized geometries of (a) $(12,0)$, (b) $(10,4)$, and (c) $(7,7)$ CNTs. Optimized geometries of (d) $(12,0),(e)(10,4)$, and (f) $(7,7) \mathrm{CNTs}$ with monovacancy. Optimized geometries of (g) $(12,0),(\mathrm{h})$ $(10,4)$, and (i) $(7,7)$ CNTs with divacancy. Red circles denote the $\mathrm{C}$ atoms surrounding the atomic defects. 


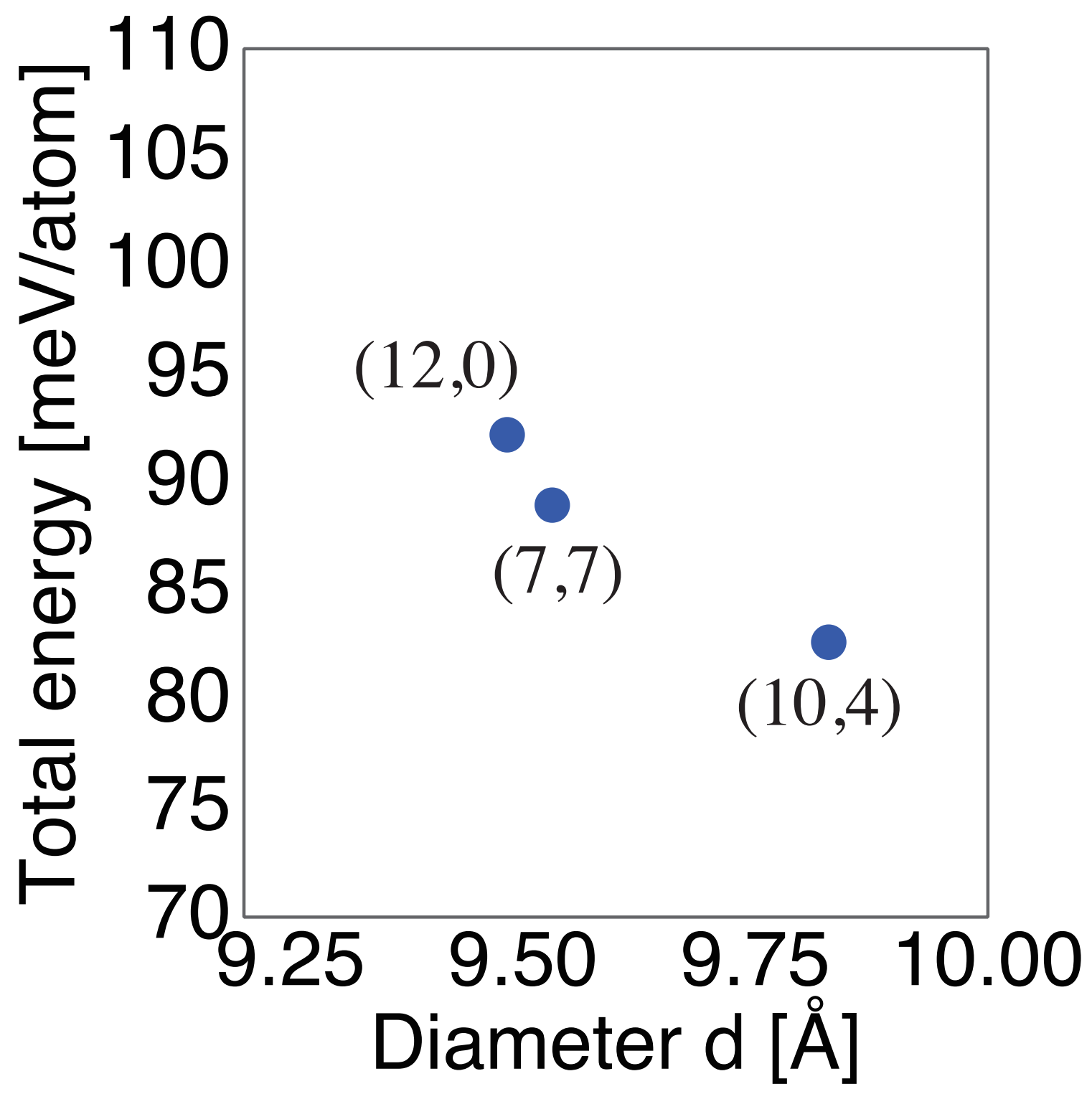

Fig. 2. The total energy per atom of $(12,0),(10,4)$, and $(7,7)$ CNTs as a function of their diameter. The energy is measured from that of graphene.

Table I. Calculated Young's modulus of $(12,0),(10,4)$, and $(7,7)$ CNTs without and with vacancies.

\begin{tabular}{cccc}
\hline CNT & \multicolumn{3}{c}{ Young's modulus (TPa) } \\
& pristine & monovacancy & divancancy \\
\hline$(12,0)$ & 0.741 & 0.670 & 0.679 \\
$(10,4)$ & 0.748 & 0.650 & 0.678 \\
$(7,7)$ & 0.754 & 0.601 & 0.695 \\
\hline
\end{tabular}


Table II. Critical fracture tensions of $(12,0),(10,4)$, and $(7,7)$ CNTs without and with vacancies. Parentheses indicate the critical tensile strain corresponding to the fracture.

\begin{tabular}{cccc}
\hline CNT & \multicolumn{3}{c}{ Critical tensile strain $(\mathrm{nN})$} \\
& pristine & monovacancy & divancancy \\
\hline$(12,0)$ & $100.1(@ 22 \%)$ & $97.7(@ 12 \%)$ & $96.6(@ 12 \%)$ \\
$(10,4)$ & $109.1(@ 24 \%)$ & $101.0(@ 22 \%)$ & $100.9(@ 12 \%)$ \\
$(7,7)$ & $99.61(@ 40 \%)$ & $88.35(@ 11 \%)$ & $100.5(@ 13 \%)$ \\
\hline
\end{tabular}

arrangement. The modulus of $(12,0),(10,4)$, and $(7,7)$ CNTs with monovacancy is 0.670 , 0650, and 0.601 TPa, respectively, which is lower than that of the pristine CNTs by $100 \mathrm{GPa}$. Interestingly, in contrast to the modulus of the pristine CNTs, the modulus of the armchair CNT with monovacancy is exceptionally lower by about $10 \%$ than those of zigzag and chiral CNTs with the monovacancy. The fact implies that the armchair CNTs with monovacancies are rarely synthesized in the mechanical views as well as its large formation energy. For the case of CNTs with divacancy, the modulus is 0.679, 0.678, and 0.695 TPa for $(12,0),(10,4)$, and (7,7)CNTs, respectively, which are lower than those of pristine CNT but almost the same as those of CNTs with monovacancy. Therefore, the modulus mainly depends on whether CNT has defect or not, but weakly depends on the defect species.

The mechanical toughness of CNTs against the tensile strain is investigated. Table II listed the critical tension of CNTs at which fracture occurs on CNTs. The critical tension weakly depends on the chirality and defects. The vacancies basically decrease the critical strain from those for the pristine CNT. $(7,7) \mathrm{CNT}$ is the mechanically toughest against the tensile strain among three pristine CNTs studied here, retaining its tubular structure up to $40 \%$ elongation. In contrast, remaining two CNTs show lower mechanical toughness in which they keep their tubular structure up to approximately $20 \%$ elongation. Vacancies substantially soften up CNTs: The critical elongation, where the CNT starts fracture, is remarkably shorter than those for pristine CNTs by about $10 \%$. On the other hand, the critical tension is almost the same as those of the pristine CNTs with the value of about $100 \mathrm{nN}$. It should be noted that $(7,7) \mathrm{CNT}$ with monovacancy is fragile against the tensile strain, owing to its energetic instability arising from an unsaturated covalent bond surrounding the defect.

Figure 3 shows the Poisson's ratio of pristine CNTs as a function of uniaxial tensile 


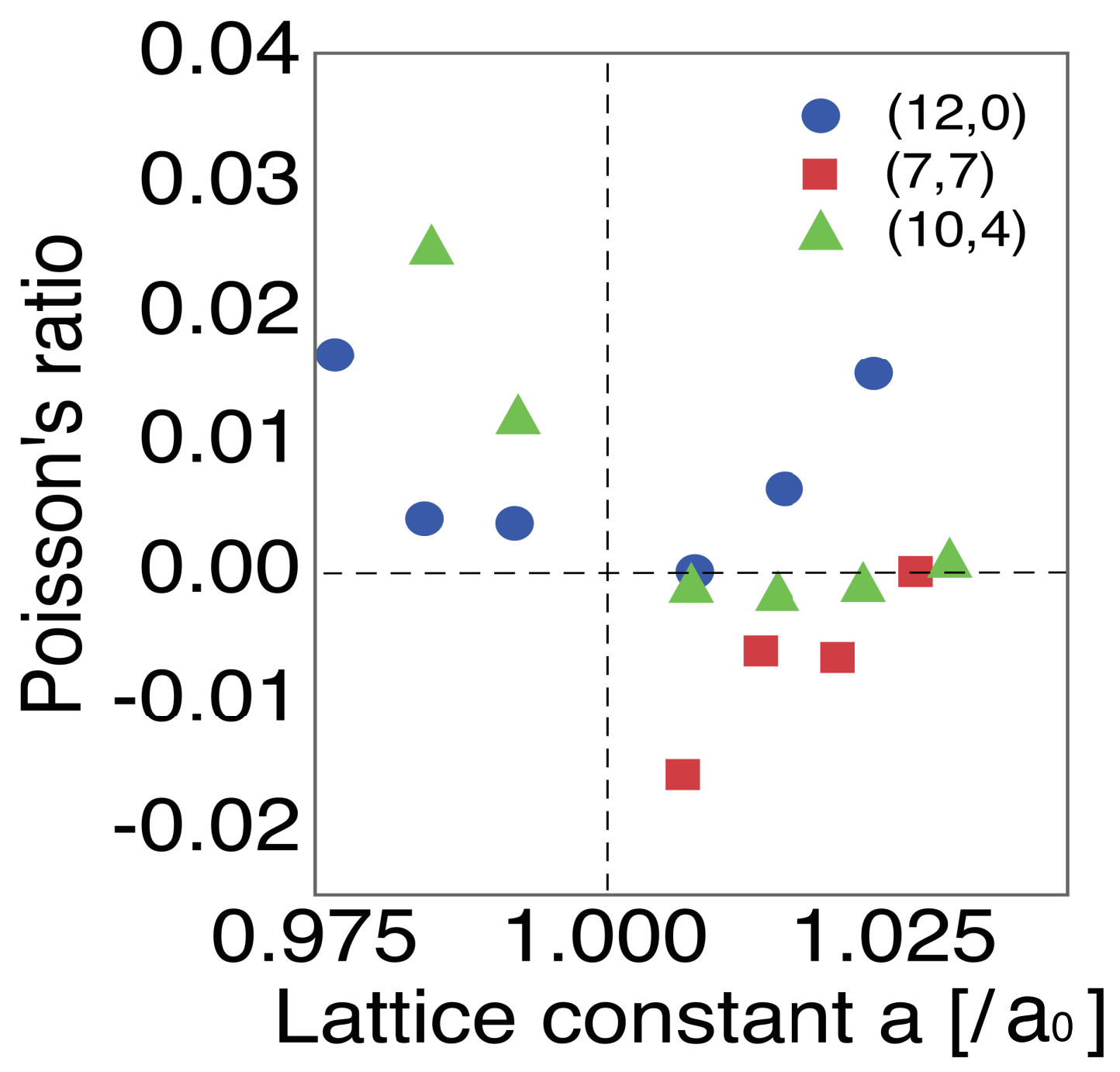

Fig. 3. Poisson's ratio of the pristine CNT as a function of uniaxial tensile strain. Circles, squares, and triangles denote the Poisson's ratio of $(12,0),(10,4)$, and $(7,7)$ CNTs, respectively.

strain. The Poisson's ratio is evaluated by taking the ratio betweens the strains along the diameter and the tube axis of CNTs. Interestingly, $(7,7)$ and $(10,4)$ CNTs possess a negative Poisson's ratio under the small tensile strain: Averaged diameters of these CNTs increase by applying the tensile strain along the tube axis. The the Poisson's ratio of $(7,7) \mathrm{CNT}$ is lower than that of $(10,4) \mathrm{CNT}$. In particular, $(7,7) \mathrm{CNT}$ shows the smallest negative Poisson's ratio under small tensile strain of $0.6 \%$. The ratio increases with increasing the tensile strain, and it crosses zero under the strain of about $2.5 \%$ for both $(7,7)$ and $(10,4)$ CNTs. By further increasing the tensile strain, these CNTs exhibit usual mechanical feature. In contrast, $(12,0) \mathrm{CNT}$ exhibits usual structural property that the diameter decrease under the tensile strain. 


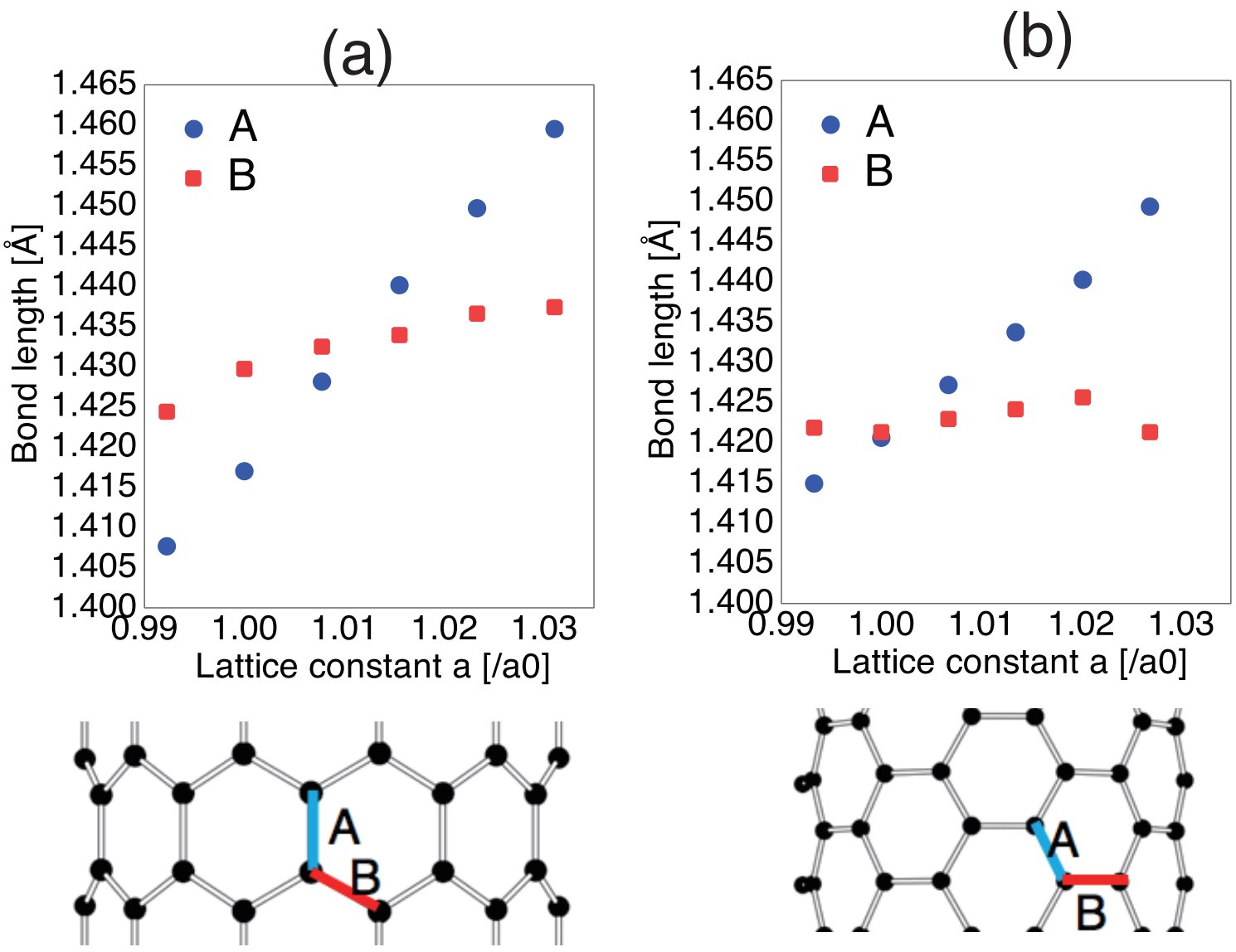

Fig. 4. The bond length of (a) $(12,0) \mathrm{CNT}$ and (b) $(7,7) \mathrm{CNT}$ as a function of the tensile strain. Circles and squares denote bonds parallel and normal, respectively, to the CNT axis as depicted in lower panels.

Finally, we discuss the physical origin of the unusual mechanical response of $(7,7) \mathrm{CNT}$ against the tensile strain. Figure 4 shows the bond length of $(12,0)$ and $(7,7) \mathrm{CNTs}$ as a function of the uniaxial compressive and tensile strains. For $(12,0) \mathrm{CNT}$, the bond along tube axis labelled $\mathrm{A}$ is rapidly elongated with increasing the tensile strain. In contrast, the bond nearly parallel to the axis of $(7,7) \mathrm{CNT}$ shows lower response to the tensile strain. The substantial elongation of the parallel bond of $(12,0) \mathrm{CNT}$ causes the small Young's modulus. For both CNTs, the bond length of the perpendicular and nearly perpendicular bonds (the bond B in Fig. 4) with respect to the axis is insensitive to the tensile. Thus, the small response of the parallel and perpendicular bonds of $(7,7)$ CNTs causes the small negative value of the Poisson's ratio under the small tensile strain. 


\section{Summary}

Using the DFT with GGA, we studied the geometric structure and the energetics of CNTs with the diameter of approximately $1 \mathrm{~nm}$ under the uniaxial tensile strain to provide the unified knowledge of the mechanical properties of CNTs in terms of their chirality and atomic defects. Our calculations demonstrated that the Young's modulus of pristine CNTs depends on their chirality: The armchair CNTs has larger Young's modulus than chiral and zigzag CNTs, and the modulus monotonically decreases with decreasing the chiral angle from armchair to zigzag. The modulus decreases by introducing atomic defects and depends on the defect size and CNT chirality. We also investigated the critical fracture tensions on these CNTs. The chiral $(10,4) \mathrm{CNT}$ is tougher against the mechanical fracture than the armchair $(7,7)$ and zigzag $(12,0)$ CNTs with similar diameter. We further found that the atomic defects substantially decrease the critical tension for all cases. As for the structural deformation, we found a small negative Poisson's ratio for armchair and chiral CNTs under the small tensile strains less than a few percent.

\section{Acknowledgements}

This work was supported by JST-CREST Grant Numbers JPMJCR1532 and JPMJCR1715 from the Japan Science and Technology Agency, JSPS KAKENHI Grant Numbers JP17H01069, JP16H00898, and JP16H06331 from the Japan Society for the Promotion of Science, and the Joint Research Program on Zero-Emission Energy Research, Institute of Advanced Energy, Kyoto University. Part of the calculations was performed on an NEC SX-Ace at the Cybermedia Center at Osaka University and on an SGI ICE XA/UV at the Institute of Solid State Physics, The University of Tokyo. 


\section{References}

1) S. Iijima, Nature 354, 56 (1991).

2) M. S. Dresselhaus, G. Dresselhaus, and P. C. Eklund: Science of Fullerenes and Carbon Nanotubes (Academic Press, San Diego, CA, 1996).

3) N. Hamada, S.-I. Sawada, and A. Oshiyama, Phys. Rev. Lett. 68, 1579 (1992).

4) R. Saito, M. Fujita, M. S. Dresselhaus, and G. Dresselhaus, Appl. Phys. Lett. 60, 2204 (1992).

5) K. Tanaka, K. Okahara, M. Okada, and T. Yamabe, Chem. Phys. Lett. 191, 469 (1992).

6) S. J. Tans, A. R. M. Verschueren, and C. Dekker, Nature 393, 49 (1998).

7) R. Martel, T. Schmidt, H. R. Shea, T. Hartel, and Ph. Avouris, Appl. Phys. Lett. 73, 2447 (1998).

8) Y. Nosho, Y. Ohno, S. Kishimoto, and T. Mizutani, Appl. Phys. Lett. 86, 073105 (2005).

9) Y. Nosho, Y. Ohno, S. Kishimoto, and T. Mizutani, Nanotechnology 17, 3412 (2006).

10) D.-M. Sun, M. Y. Timmermans, Y. Tian, A. G. Nasibulin, E. I. Kauppinen, S. Kishimoto, T. Mizutani, and Y. Ohno, Nat. Nanotechnol. 6, 156 (2011).

11) R. S. Ruoff and D. C. Lorents, Carbon 33, 925 (1995).

12) M. M. J. Treacy, T. W. Ebbesen, and J. M. Gibson, Nature 381, 678 (1996).

13) S. Berber, Y.-K. Kwon, and D. Tománek, Phys. Rev. Lett. 84, 4613 (2000).

14) T. Yamamoto, S. Watanabe, and K. Watanabe, Phys. Rev. Lett. 92, 075502 (2004).

15) B. W. Smith, M. Monthioux, and D. E. Luzzi, Nature 396, 323 (1998).

16) K. Hirahara, K. Suenaga, S. Bandow, H. Kato, T. Okazaki, H. Shinohara, and S. Iijima, Phys. Rev. Lett. 85, 5384 (2000).

17) S. Okada, S. Saito, and A. Oshiyama, Phys. Rev. Lett. 86, 3835 (2001).

18) T. Okazaki, Y. Iizumi, S. Okubo, H. Kataura, Z. Liu, K. Suenaga, Y. Tahara, M. Yudasaka, S. Okada, and S. Iijima, Angew. Chem. Int. Ed. 50, 4853 (2011).

19) M. Fujihara, Y. Miyata, R. Kitaura, Y. Nishimura, C. Camacho, S. Irle, Y. Izumi, T. Okazaki, and H. Shinohara, J. Phys. Chem. C 116, 15141 (2012).

20) S. Kigure, Y. Iizumi, T. Okazaki, and S. Okada, J. Phys. Soc. Jpn. 83, 124709 (2014). 
21) H. E. Lim, Y. Miyata, M. Fujihara, S. Okada, H. Omachi, R. Kitaura, and H. Shinohara, ACS NANO 9, 5034 (2015).

22) T. Koyama, K. Fujiki, Y. Nagasawa, S. Okada, K. Asaka, Y. Saito, and H. Kishida, J. Phys. Chem. C 122, 5805 (2018).

23) A. Takakura, K. Beppu, T. Nishihara, A. Fukui, T. Kozeki, T. Namazu, Y. Miyauchi, and K. Itami, Nat. Commun. 10, 3040 (2019).

24) G. Overney, W. Zhong, D. Tománek, Z. Phys. 27, 93 (1993).

25) T. Ozaki, Y. Iwasa, and T. Mitani, Phys. Rev. Lett. 84, 1712 (2000).

26) Q. Zhao, M. B. Nardelli, and J. Bernholc Phys. Rev. B 65, 144105 (2002)

27) S. Ogata and Y. Shibutani, Phys. Rev. B 68, 165409 (2003).

28) M. Sammalkorpi, A. Krasheninnikov, A. Kuronen, K. Nordlund, and K. Kaski, Phys. Rev. B 70, 245416 (2004).

29) H. Mori, Y. Hirai, S. Ogata, S. Akita, and Y. Nakayama, Jpn. J. Appl. Phys. 44, L1307 (2005).

30) P. Hohenberg and W. Kohn, Phys. Rev. 136, B864 (1964).

31) W. Kohn and L. J. Sham, Phys. Rev. 140, A1144 (1965).

32) Y. Morikawa, K. Iwata, and K. Terakura, Appl. Surf. Sci. 169-170, 11 (2001).

33) J. P. Perdew, K. Burke, and M. Ernzerhof, Phys. Rev. Lett. 77, 3865 (1997).

34) J. P. Perdew, K. Burke, and M. Ernzerhof, Phys. Rev. Lett. 78, 1396 (1997).

35) D. Vanderbilt, Phys. Rev. B 41, 7892 (1990).

36) M. Maruyama and S. Okada, Appl. Phys. Express 9, 095101 (2013). 